\title{
Фотоэлектрические свойства нитевидных кристаллов ZnO
}

\author{
() П.С. Шкумбатюк
}

Дрогобычский государственный педагогический университет им.И. Франко, 82100 Дрогобыч, Украина

E-mail: tarasik82@gmail.com

(Получена 7 марта 2017г. Принята к печати 5 апреля 2017г.)

Исследованы фотоэлектрические свойства нитевидных кристаллов $\mathrm{ZnO}$, полученных из газовой фазы испарением порошка и металлического $\mathrm{Zn}$ непрерывным действием излучения $\mathrm{CO}_{2}$-лазера. На основании исследования баръерной структуры $\mathrm{InO}-\mathrm{ZnO}$ предложен механизм фотопроводимости кристаллического $\mathrm{ZnO}$, обусловленный потенциальными барьерами.

DOI: $10.21883 /$ FTP.2018.07.46045.8576

\section{1. Введение}

Оксид цинка - известный полупроводниковый материал фоточувствительный в ультрафиолетовой области спектра [1]. В настоящее время применение этого свойства ограничено большим временем релаксации и нестабильностью фотопроводимости. Возможно, это связано не с химическими и физическими свойствами $\mathrm{ZnO}$, а с технологией его получения и легирования.

При общих направлениях изучения фотоэлектрических свойств $\mathrm{ZnO}$ используют результаты исследования спектральной характеристики, пороговой чувствительности, кинетики релаксации фотоотклика. Для обяснения фотоэлектрических свойств кристаллического $\mathrm{ZnO}$ привлекаются механизмы изменения поверхностных свойств под действием ультрафиолетового (УФ) излучения, свойств поверхностей между кристаллитами, влияние собственных структурных дефектов, примесей металлов, внешних условий $[2,3]$.

Важное направление в поисках возможного получения качественных фотоприемников занимает исследование зависимости указанных свойств от технологии выращивания $\mathrm{ZnO}[4,5]$. С этой целью в данной работе для получения кристаллического $\mathrm{ZnO}$ с паровой фазы использовали тепловое действие непрерывного излучения $\mathrm{CO}_{2}$-лазера.

\section{2. Эксперимент}

Нитевидные монокристаллы $\mathrm{ZnO}$ (рис. 1) были получены испарением порошка $\mathrm{ZnO}$, поликристаллические - окислением $\mathrm{Zn}$ [6]. Размеры полученных нитей: диаметр $(d)$ 2-6 мкм, длина $(l) 0.6-0.8$ мм для монокристаллов и $d=10-50$ мкм, $l=0.6-0.8$ мм для поликристаллов. Образцы устанавливали на сапфировые подложки. Для исследования фотоэлектрических свойств полученного $\mathrm{ZnO}$ измерялись вольт-амперные характеристики $(\mathrm{BAX})$ при напряжении $V=0-4 \mathrm{~B}$, спектральная зависимость фотопроводимости, постоянная времени фотоответа и температурная зависимость проводимости в области температур $T=300-450 \mathrm{~K}$ на воздухе и в вакууме. Для исследования влияния
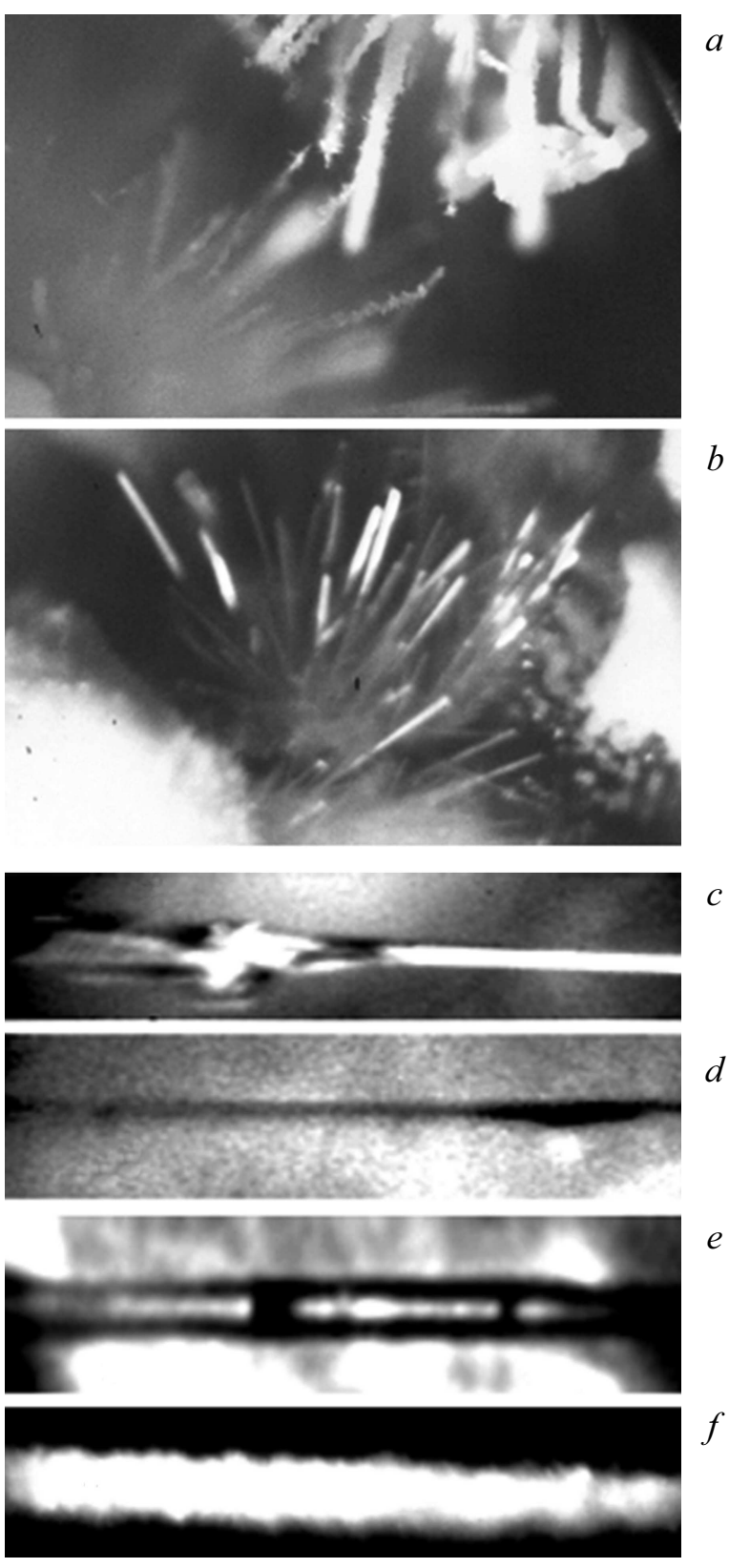

Рис. 1. Фотография нитевидных кристаллов $\mathrm{ZnO}: a-$ поликристаллический, $b-$ монокристаллический, $c-f-$ фоточувствительный. 
контактов использовались прижимные и вплавленные контакты In, Ga, и контакты Al, Zn, напиленные термическим методом в вакууме $2-5 \cdot 10^{-4}$ мм. рт. ст. При изготовлении барьерных структур металл-полупроводник был использован In, окисленный при $T=800-900 \mathrm{~K}$, в течение $t=20-60$ с. Для анализа электрических свойств приконтактной области $\mathrm{InO}-\mathrm{ZnO}$ на воздухе и в вакууме исследовались переходные процессы переключения полярности напряжения и влияние ультрафиолетового излучения. До изготовления контактов образцы на подложках прогревали на воздухе при $T=800-900 \mathrm{~K}$, время нагревания 10-20 с. Вплавление In и Ga производилось в вакууме и на воздухе при $T=450-800 \mathrm{~K}$. Из сравнения ВАХ до и после последовательных двухтрех нагреваний и охлаждений при $T=450-300 \mathrm{~K}$ (термоциклирование) в нормальных условиях определялось влияние температуры на свойства контактов. Спектральная зависимость фотопроводимости измерялась с помощью усилителя У5-8 на монохроматоре ДМР4 (ширина щели была 0.4 мм), лампа была КГМ-250 (ток накала 7 А). Мощность излучения в ультрафиолетовой области была измерена с помощью фотоэлемента Ф-16, (для $\lambda=380$ нм она составляла $P=2-4 \cdot 10^{-7}$ Вт). При измерении постоянной времени фотоответа и переходных процессов использовался самописец с чувствительностью 25 мкВ/см, входное сопротивление 75 Ом, фильтр $\mathrm{CC}-14$ толщиной 2 мм, ток накала $5 \mathrm{~A}$, расстояние до образца $25 \mathrm{~cm}$. Температурная зависимость проводимости (ТЗП) была измерена в двух направлениях тока в криостате в нормальных условиях и в вакууме $2-3 \cdot 10^{-2}$ мм рт. ст. (термопара медь-константан, усилитель У1-7 или самописец при постоянном напряжении $V=1-2$ В). Для изменения концентрации структурных дефектов образцы отжигали на сапфировых подложках в области температур 700-1100 K на протяжении 0.6-8 ч на воздухе.

\section{3. Результаты и обсуждение}

Вольт-амперные характеристики $\mathrm{ZnO}$ с In, Ga, Zn, $\mathrm{Al}$ контактами в вакууме и на воздухе при $300 \mathrm{~K}$ в прямом и обратном направлении для моно- и поликристаллического $\mathrm{ZnO}$ были линейные и симметричные. Нагрев и охлаждение $\mathrm{ZnO}$ с In-, Ga-контактами на воздухе в диапазоне температур $300-450 \mathrm{~K}$ увеличивают контактное сопротивление $\mathrm{Ga}-\mathrm{ZnO}$. После термоциклирования $\mathrm{ZnO}$ с Zn-контактами BAX восстанавливаются до исходных на протяжении 1-3 сут. с возможным появлением нестабильности тока (низкочастотный шум). При переключении направления тока с Al-контактами в электрическом поле в области $10^{3} \mathrm{~B} / \mathrm{cm}$ происходит разрушение $\mathrm{Al}-$ контактов на границе $\mathrm{ZnO}-\mathrm{Al}$ в виде разрыва или удаление частей Al-пленки. Нагрев в вакууме или на воздухе до $450 \mathrm{~K}$ ВАХ (см. вставку к рис. 2, кривые 3,4$)$ уменьшает контактное сопротивле-

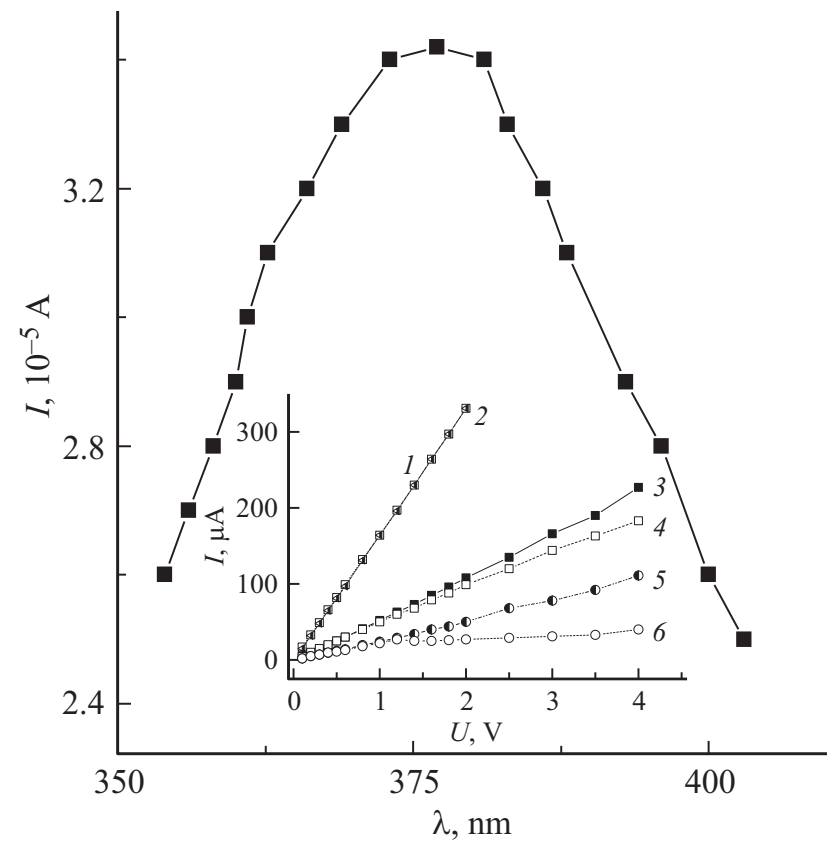

Рис. 2. Спектральная зависимость фотопроводимости $\mathrm{ZnO}$. На вставке - BAX ZnO In-InO-контактами: 1,3,5 - положительная полярность на InO, 2,4,6 - отрицательная полярность на $\mathrm{InO}$.

ние $\mathrm{InO}-\mathrm{ZnO}$ и нелинейность ВАX (см. вставку к рис. 2, кривые 1,2).

Фотопроводимость монокристаллического $\mathrm{ZnO}$ с линейными и симметричными ВАХ исследованных контактов, при $300 \mathrm{~K}$ на воздухе и вакууме не наблюдалась, за исключением $\mathrm{ZnO}$, показанного на рис. $1, c-e$. Спектральная зависимость фотопроводимости фоточувствительного монокристаллического $\mathrm{ZnO}$ на воздухе и в вакууме показана на рис. 2. Поликристаллический $\mathrm{ZnO}$ имеет второй максимум в области 0.5 мкм. При хранении фоточувствительного $\mathrm{ZnO}$ в нормальных условиях наблюдали уменьшение фотопроводимости, нагревание $\mathrm{ZnO}$ до 400-600 K восстанавливает фотопроводимость. Фотоочувствление отожженного монокристаллического $\mathrm{ZnO}$ не наблюдали. Для поликристаллического $\mathrm{ZnO}$ наблюдали уменьшение фотопроводимости в ультрафиолетовой области и увеличение в видимой области спектра. Временные зависимости увеличения фотопроводимости и темновой релаксации исследуемого $\mathrm{ZnO}$ имеют две составляющие и зависят от внешних условий (рис. 3, $a$ и $b$ ).

При исследовании температурной проводимости в вакууме на кривых ТЗП при охлаждении наблюдали увеличение проводимости (рис. 3, $a, b$, кривая 2). Повторный нагрев в вакууме монокристаллического $\mathrm{ZnO}$ до $350-370 \mathrm{~K}$ восстанавливает начальную темновую проводимость. Повторный нагрев в вакууме до $450 \mathrm{~K}$ поликристаллического $\mathrm{ZnO}$ не восстанавливает начальную проводимость. Температурная зависимость прово- 


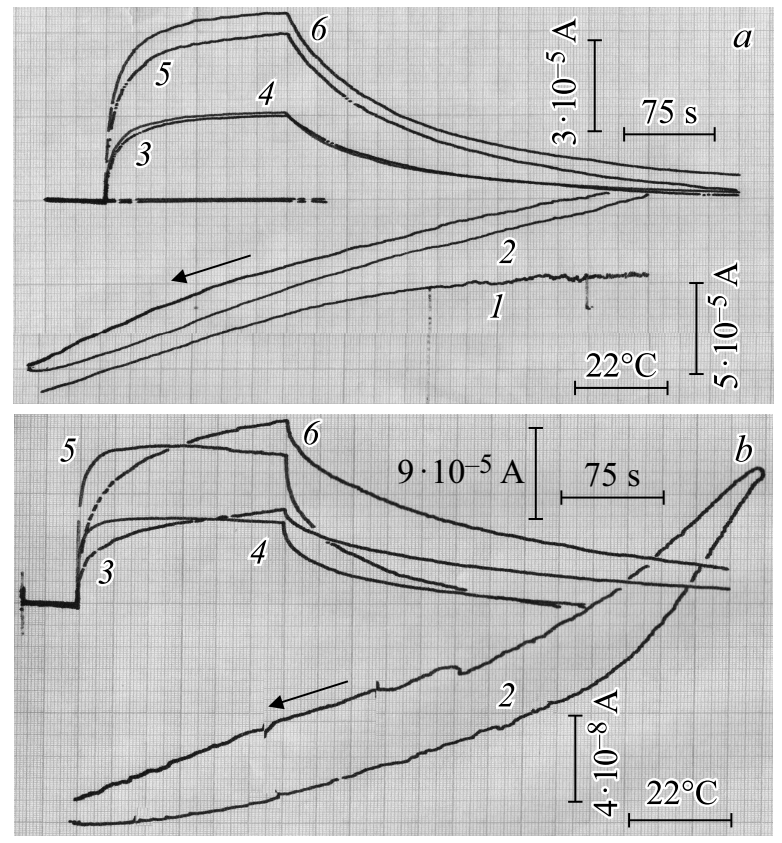

Pис. 3. Температурная зависимость проводимости монокристаллического $(a)$ и поликристаллического $(b) \mathrm{ZnO}: 1-$ на воздухе, $2-$ в вакууме (стрелка - охлаждение); временна́я зависимость увеличения фотопроводимости и темновой релаксации монокристаллического $(a)$ и поликристаллического $(b)$ $\mathrm{ZnO}$ : при $V=1$ В на воздухе (3) и в вакууме $(4)$, при $V=2 \mathrm{~B}$ на воздухе (5) и в вакууме (6).

димости монокристаллического не фоточувствительного $\mathrm{ZnO}$ отсутствует.

Кривые ТЗП можно отобразить аналитически зависимостью вида

$$
\ln y=-(x)^{k} \ln a,
$$

где $y$ - изменение концентрации носителей заряда от температуры, $a, k$ - произвольные константы $x=E_{d} / k T, E_{d}-$ энергия активации дефекта. Удовлетворительная аналитическая аппроксимация, за исключением конечных значений $I=f(T)$ для кривой 2 рис. 3, $a$, показана на рис. 4. Механизм несовпадения ТЗП при нагревании и охлаждении в вакууме кристаллического $\mathrm{ZnO}$ не исследовали.

Проведенные исследования показали, что фотоэлектрические свойства кристаллического $\mathrm{ZnO}$ зависят от распределения структурных дефектов с возможным образованием потенциальных барьеров. Для исследования предполагаемой модели использовали нефоточувствительный монокристаллический $\mathrm{ZnO}$ с $\mathrm{In}-\mathrm{InO}$ контактами. Результат влияния ультрафиолетового излучения показан на рис. 5, $a$ (кривые 2,3).

Для анализа вероятности изменения сопротивления контакта $\mathrm{InO}-\mathrm{ZnO}$ при облучении ультрафиолетовым излучением исследовали МДП-структуры In, InOслюда толщиной 20 мкм - нефоточувствительный $\mathrm{ZnO}$ In-контакт. На рис. 5, a (кривая 4) показана фотопрово- димость в ультрафиолетовой области МДП-структуры в нормальных условиях. При положительной полярности напряжения на контакте InO-слюда в видимой области спектра, в среде кислорода, водорода и в вакууме фотопроводимость не наблюдали. Короткодействующая (емкостная) составляющая обусловлена десорбцией гид-

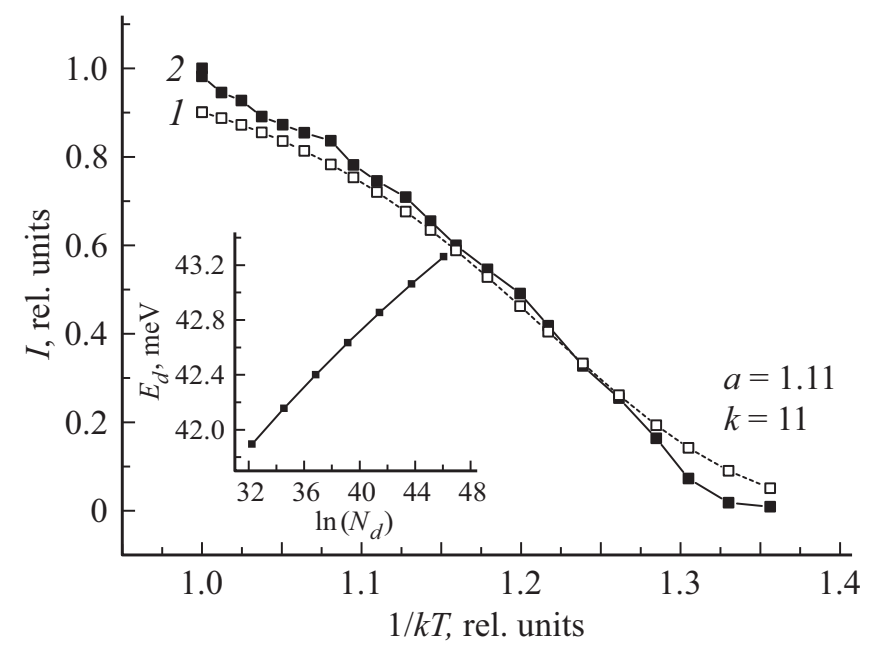

Рис. 4. Аналитическая аппроксимация (кривая 1) температурной зависимости проводимости монокристаллического $\mathrm{ZnO}$ (кривая 2). На вставке - зависимость $E_{d}$ от концентрации носителей заряда для кривой 1.

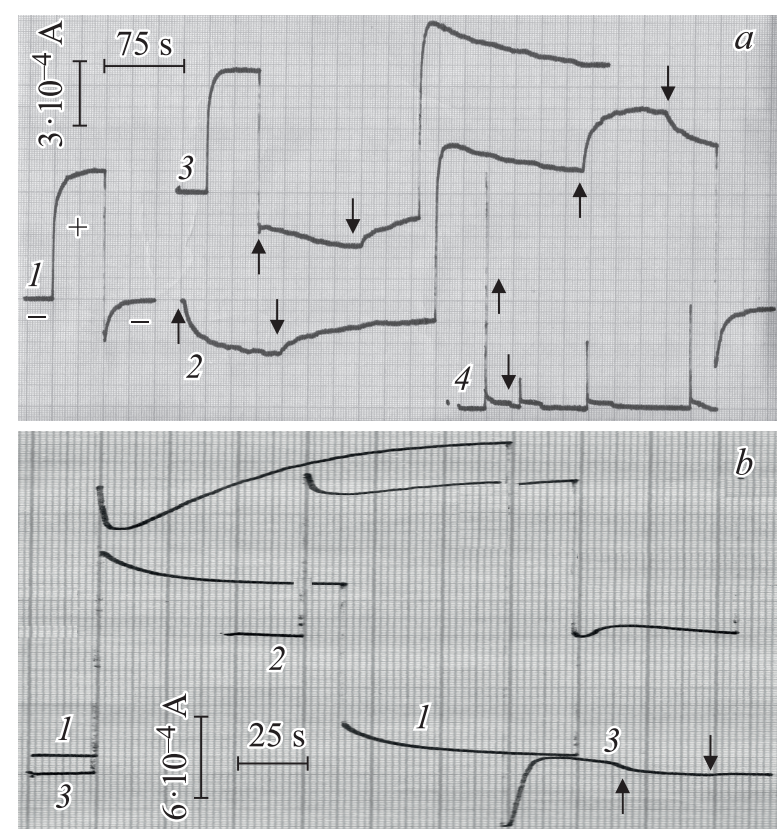

Рис. 5. Переходные процессы на контакте InO-ZnO: нефоточувствительного (a) и дефектного $(b) \mathrm{ZnO}$. (a) 1 - кривая переключения полярности напряжения на InO, 2 и 3 - облучение ультрафиолетовым излучением, $4-$ фотоотклик МДПструктуры при облучении ультрафиолетовым излучением, на слюде $V=-80 \mathrm{~B}$. (b) $1-3-V_{3}>V_{2}>V_{1} ; 3-$ облучение ультрафиолетовым излучением. Стрелка вверх - включение света, стрелка вниз - выключение света. 
ратированной воды, захваченной положительно заряженными дефектами. Величина тока последующих импульсов фотоотклика зависит от времени между освещением и указывает на долговременный процесс заполнения дефектов. Независимость постоянной составляющей фотопроводимости от степени заполнения дефектов указывает на влияние адсорбированной воды между контактами, включая перезарядку дефектов на контакте слюда- $\mathrm{ZnO}$. Отсутствие фоточувствительности при положительной полярности напряжения обусловлено экранированием электрического поля на контакте $\mathrm{InO}-$ слюда. Изменение вида кривых фотоотклика при использовании контакта In-слюда не наблюдали. Из приведенного анализа МДП-структуры следует, что влияние ультрафиолетового излучения обусловлено изменением электрических свойств приконтактной области $\mathrm{InO}-\mathrm{ZnO}$.

На рис. 5, а показаны переходные процессы при переключении полярности напряжения и облучении ультрафиолетовым излучением $\mathrm{InO}-\mathrm{ZnO}$ со стороны $\mathrm{ZnO}$. Время опустошения и заполнения положительно заряженных дефектов в подконтактной области (рис. 5, $a$, кривая 1) зависит от сопротивления контакта, концентрации уровней прилипания и напряжения. При освещении InO-ZnO с отрицательной полярностью напряжения (рис. 5, $a$, кривые 2,3 ) наблюдали добавочную фотопроводимость со временем нарастания больше времени перезарядки дефектов, обусловленную фотоэмиссией носителей заряда с $\mathrm{InO}$ в $\mathrm{ZnO}$ [7]. При положительной полярности (рис. 5, a, кривая 2) наблюдали инжекцию дырок в подконтактную область $\mathrm{InO}-\mathrm{ZnO}$ и уменьшение времени релаксации при отрицательной полярности напряжения. Сравнение нарастания и релаксации при отрицательной и положительной полярности напряжения указывает на влияние потенциальных барьеров на кинетику фотопроводимости $\mathrm{InO}-\mathrm{ZnO}$ [8]. Переходные процессы дефектного $\mathrm{ZnO}$ с $\mathrm{InO}-$-контактом показаны на рис. $5, b$. При напряжении $0.5-1 \mathrm{~B}$ и положительной полярности на InO (рис. 2, кривые 5,6) происходит изменение зарядового состояния дефектной области $\mathrm{ZnO}$ (рис. 5, $b$, кривая 1 ), при увеличении напряжения (рис. 5, $b$, кривые 2,3) происходит изменение зарядового состояния в приконтактой области $\mathrm{InO}-\mathrm{ZnO}$ и фотоочувствление дефектного $\mathrm{ZnO}$ с InO-контактом в видимой области спектра.

Результаты исследования $\mathrm{InO}-\mathrm{ZnO}$ позволяют сделать вывод о том, что механизм наблюдаемой фоточувствительности кристаллического $\mathrm{ZnO}$ с омическими контактами обусловлен потенциальными барьерами. Полученные данные не позволяют определить природу дефектов в исследуемом кристаллическом ZnO. Определенная по ТЗП тепловая энергия ионизации дефектов (обсуждалась в работе [9]) обусловлена структурными дефектами и отвечает донорному уровню. По результатам исследования барьерного контакта In- ZnO можно только предположить, что поверхность монокристаллического $\mathrm{ZnO}$ обеднена основными носителями заряда.

\section{4. Заключение}

Исследование фотоэлектрических свойств нитевидных кристаллов $\mathrm{ZnO}$, полученных из паровой фазы действием излучения $\mathrm{CO}_{2}$-лазера, показало, что механизм изменения проводимости под действием УФ излучения связан с потенциальными барьерами. Вывод о механизме фотопроводимости сделан на основании исследования барьерной структуры $\mathrm{InO}-\mathrm{ZnO}$ не фоточувствительного $\mathrm{ZnO}$. Фотоэлектрические свойства $\mathrm{ZnO}$ под действием барьерного механизма связаны с большим временем нарастания фотопроводимости и темновой релаксацией. Отметим, что возможность управлять использованной технологией для получения фоточувствительного монокристаллического $\mathrm{ZnO}$ не выявлена.

\section{Список литературы}

[1] Б. Гудден. УФН, 15 (6), 703 (1935).

[2] R.J. Collins, D.G. Thomas. Phys. Rev., 112, (2), 388 (1958).

[3] G. Chai, O. Lupan, L. Chow, H. Heinrich. Sensors Actuators A, 150, 184 (2009).

[4] R. Ayouchi, L. Bentes, C. Casteleiro, O. Conde, C.P. Marques, E. Alves, A.M.C. Moutinho, H.P. Marques, O. Teodoro, R. Schwarz. Appl. Surf. Sci., 255, 5917 (2009).

[5] В.А. Кривченко, Д.В. Лопаев, П.В. Пащенко, В.Г. Пирогов, А.Т. Рахимов, Н.В. Суетин, А.С. Трифонов. ЖТФ, $78(8), 107$ (2008).

[6] П.С. Шкумбатюк. ФТП, 44 (8), 1147 (2010).

[7] С.М. Зи. Физика полупроводниковых приборов (М., Мир, 1984).

[8] М.К. Шейнкман, А.Я. Шик. ФТП, 10 (2), 209 (1976).

[9] A.R. Hutson. Phys. Rev., 108 (2), 222 (1957).

Редактор А.Н. Смирнов

\section{Photo-electric properties of threadlike srystals $\mathrm{ZnO}$}

P.S. Shkumbatjuk

State pedagogical university of I. Franko, 82100 Drogobych, Ukraine

Abstract Photo-electric properties of threadlike crystals $\mathrm{ZnO}$ received with a gas phase evaporation of a powder and metal $\mathrm{Zn}$ continuous action of radiation $\mathrm{CO}_{2}$-laser are investigated. On the basis of research barrier structures $\mathrm{InO}-\mathrm{ZnO}$ the mechanism of photoconductivity crystal $\mathrm{ZnO}$ is conditioned space charge barrier. 